\title{
Usability of Lightweight Defibrillators for UAV Delivery
}

\section{Mathias Fleck}

Senior User Experience Researcher

Google X

Mountain View, CA 94043, USA

msfleck@gmail.com
Permission to make digital or hard copies of part or all of this work for personal or classroom use is granted without fee provided that copies are not made or distributed for profit or commercial advantage and that copies bear this notice and the full citation on the first page. Copyrights for thirdparty components of this work must be honored. For all other uses, contact the Owner/Author.

Copyright is held by the owner/author(s).

CHI'16 Extended Abstracts, May 07-12, 2016, San Jose, CA, USA

ACM $978-1-4503-4082-3 / 16 / 05$.

http://dx.doi.org/10.1145/2851581.2892288

\begin{abstract}
This case study describes a usability assessment of lightweight automated external defibrillators (AEDs) in an exploration of how AEDs might safely integrate within an unmanned aerial vehicle (UAV) delivery system to rapidly treat victims of cardiac arrest. Untrained laypersons were asked to use an AED in a simulated cardiac arrest scenario in either standard or UAV-delivery scenarios. The impact of device-specific customization of emergency operator instruction was also evaluated.
\end{abstract}

\section{Author Keywords}

Usability; automated external defibrillator (AED); delivery; unmanned aerial vehicle (UAV); drone; Schiller; HeartSine

\section{ACM Classification Keywords}

H.5.m. Information interfaces and presentation (e.g. $\mathrm{HCI})$ : Miscellaneous.

\section{Introduction}

Sudden Cardiac Arrest (SCA) is a leading cause of death in adults in the United States [5]. It is a condition in which heart activity suddenly stops, and treatment with cardiopulmonary resuscitation (CPR) and an automated external defibrillator (AED) is needed within minutes for the patient to live. Less than $8 \%$ of people 


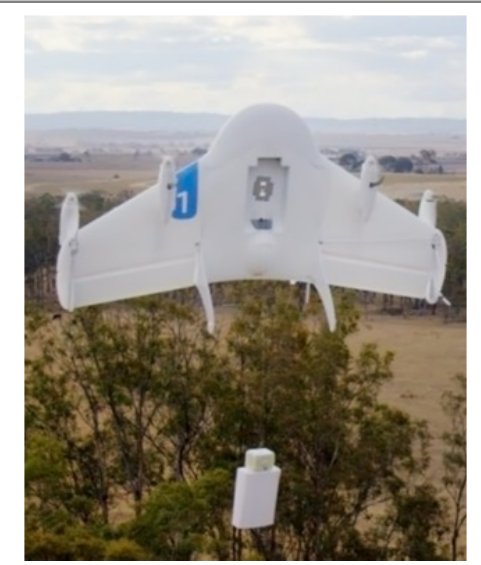

Figure 1. A Project Wing unmanned aerial vehicle (UAV) prototype demonstrating a small package delivery in August 2014 suffering SCA outside of a hospital survive the episode. [1]

Technological evolutions in emergency medical response present new scenarios and opportunities for treating SCA. Google[x], a Google lab that aims for moonshots in technology and science, recently initiated Project Wing (see Figure 1) and explored the possibility of transporting AEDs by unmanned aerial vehicle (UAV) to victims of cardiac arrest in order to provide rapid, life-saving treatment. The survival rates for defibrillation are directly tied to how quickly the AED is administered; thus, the rapidity of a small, light delivery aircraft could be a critical advantage.

However, new scenarios like these may introduce unanticipated requirements and impact the usability of medical devices. Our goal was to measure how the usability of "flying defibrillators" would impact mission success rates.

\section{Background on Use Case}

Initial modeling and research revealed that rapidly delivering defibrillators by UAV could save many lives: Every minute lapsed after cardiac arrest before receiving a shock results in a $7-10 \%$ decrease in survival rate [5]. Unfortunately, in many rural or congested regions of the country and the world, ambulances can take more than 10 minutes to arrive with the life-saving defibrillator. A UAV could rapidly fly similar distances in much faster times, potentially leading to drastically improved outcomes.

However, defibrillators are often fairly heavy, packing a large battery in order to remain functional through many months of disuse. They are typically designed to be mounted on the walls of public areas for long periods of time, rather than optimizing for weight and flight.

The Project Wing engineering team specified that the delivered payload must be small and light enough to safely accommodate aerodynamic constraints and battery/range limitations, which made many defibrillator options not viable for the project due to size. Therefore, it was important to establish how the lightest defibrillators would fare in usability studies.

\section{Previous Research}

Successfully using a defibrillator is a complicated, multi-step process, and few laypersons possess training in AED use, which places an imperative on providing clear instructions to the untrained user. The situation may be further complicated by the emotional panic and confusion present during cardiac arrests.

Earlier human factors research has demonstrated that different models of defibrillators yield varying performance in layperson use $[2,3,4,6]$. Depending on the device, typical errors observed include: failing to perform CPR or providing low-quality CPR, failure to turn AED devices on, improper electrode pad placement, and lengthy delays in defibrillation administration.

Usability affordances vary by defibrillator model and feature set; some models provide stepwise voice instruction, whereas others use illustrations to walk a user through the process. Some units have preconnected electrodes and/or automatically power on, whereas others come with detached pads or have manual power switches. The presence or absence of 
various features has been shown to impact success rates and time-to-shock [6].

Previous research has not directly explored the impact of emergency operator assistance on the usability of various AED models, which may improve success rates over that observed in typical unassisted studies.

Additionally, no studies have simulated the scenario of having a defibrillator delivered to their location; previous studies have typically begun with the AED present and near a mannequin representing the cardiac arrest victim. The present research aimed to explore these topics as well as establish baseline success rates for small, light, "UAV-friendly" defibrillators.

\section{User Studies}

I identified the two lightest weight defibrillators on the market in the U.S. at the time of the exploration (March 2013): the Schiller FRED EasyPort (600 grams) and the HeartSine samaritan PAD 300P (1100 grams) (see Figure 2). The former model is not designed for layperson use, but is far and away the lightest defibrillator available. I brought trainer versions of these two AED models into the lab to evaluate basic, unassisted usability as well as usability with assistance, including operator guidance during a simulated UAVdelivery scenario.

In Study 1, 28 adults volunteered to participate in an emergency simulation. Older participants (average age $=48$ ) without medical training were selected to best represent the likely demographic of potential at-home witnesses to cardiac arrest. Participants were instructed to rush into a room and administer defibrillation to a mannequin representing a victim of cardiac arrest.
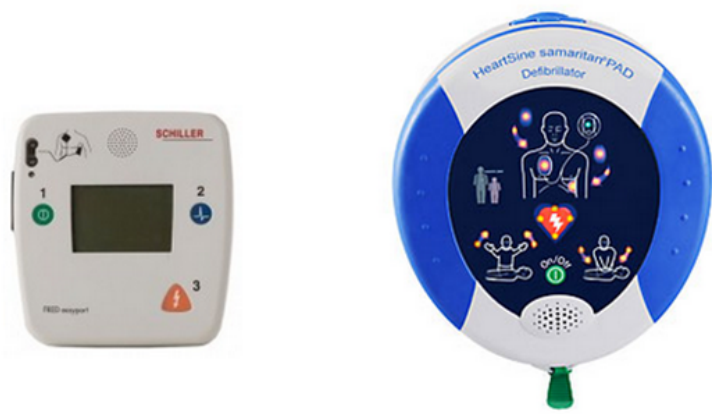

Figure 2. Left: Schiller FRED EasyPort; Right: HeartSine samaritan PAD 300P.

Participants were randomly assigned to use either the Schiller $(n=15)$ or the HeartSine $(n=13)$ AED located within the room. The mannequin (Laerdal Resusci Anne) was hooked up to a device to quantitatively assess CPR quality (Laerdal SkillTrainer). Measures collected included: success of delivering shock, time-toshock, accuracy of pad placement, and quality of CPR.

For the Schiller unit, 6 out of 15 participants (40\%) failed to successfully deliver a shock (Figure 3 ). The primary reason for failure-to-shock was due to not removing the victim's clothing before applying the electrodes. These participants failed to notice the first step represented pictorially on the packaging, showing a shirt being removed from the patient. Even for participants who successfully completed a shock, usability-related confusion added an average of 61 seconds of delay to the overall time required to administer the AED, primarily due to difficulty plugging in the electrodes. As a reminder, each minute of delay in administering a shock leads to a $7-10 \%$ decrease in survival rate. Additionally, $60 \%$ of participants placed pads in the incorrect location. $100 \%$ of participants 
performed poor CPR, as the AED guidance offered only minimal instructions about how to do so.
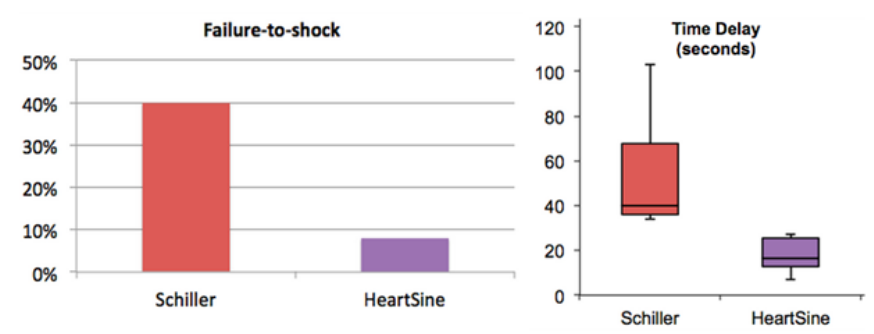

Figure 3. Usability issues impact shock success and time taken.

For the Heartsine unit, performance was much better: only $8 \%$ of participants ( 1 out of $13 ; p=.05$ ) failed to deliver a shock, and usability problems added on average 22 seconds delay, better than the Schiller $(p=.02) .15 \%$ of participants placed pads in the incorrect locations. The improved performance was driven by more detailed verbal guidance from the unit, more detailed pictorial representation of steps, and electrodes being pre-attached to the AED. CPR was still relatively low quality across participants, although it was somewhat improved due to more verbal guidance and an audible metronome for chest compressions. Overall, although user performance was much better for the HeartSine unit, the increased weight of the device (nearly double the Schiller unit) represented a much greater flight-engineering challenge, and so I explored additional ways to mitigate errors with the lightweight Schiller AED.

Because the errors for the Schiller unit were driven by failure to follow provided instructions, especially around removing the victim's clothing, I conducted a follow-up study to determine if simple, pre-task instructions might improve performance. In Study 2, I used a similar paradigm for 17 participants with the Schiller AED, but this time gave them 2 printed pieces of instruction before the study, to simulate what might be added to the side of the UAV package or otherwise provided before AED arrival: "1) Remove clothing to expose chest. 2) Turn unit on and listen to instructions." With this small change, only $24 \%$ of participants failed to deliver a shock, representing a small improvement over Study 1 (Figure $4 ; p=.16$ ), but still exhibiting some key user errors. A high number of participants (47\%) continued to place pads improperly in Study 2, which was expected because the provided instructions did not target that particular error type.

Finally, I wanted to determine if an emergency operator might be able to improve user performance with the Schiller AED. A likely UAV user scenario would involve a caller calling emergency services, who could deploy an aircraft carrying a specific model of AED. Therefore, an operator could potentially have device-specific training to identify and assist with any usability issues encountered. To test this, I visited a local 9-1-1 emergency call center and learned the script and process used by operators during cardiac arrest events. Note that typical SCA scripts have very generic language around advising callers to use AEDs, generalized to accommodate the vast diversity of models on the market. I explored the hypothesis that an operator deploying a custom UAV response unit may have customized scripts since they could know exactly which AED the user would be administering and could potentially even hear device audio cues to know how best to assist the caller. 
In Study 3, 15 older adult participants (average age $=51$ ) volunteered to participate in an emergency simulation using the Schiller AED in which they were asked to first call "emergency services" upon entering the room. I played the role of an emergency operator, directing participants by telephone on how to begin CPR and later run to retrieve an AED unit "delivered" outside the room. I followed the standard cardiac arrest script with minor changes to provide Schiller-specific details in order to assist the caller. I also listened for AED audio cues to understand where the user might be stuck, and corrected mistakes that I could hear through the phone. With this simulated 9-1-1 guidance,

performance dramatically improved: $0 \%$ of participants failed to deliver a shock, representing a significant improvement over Study $1(p<.001)$. Usability-related time-to-shock delays were reduced to 16 seconds, significantly less than in Study $1(p<.001)$. Only 2 out 15 participants (13\%) placed electrodes improperly. The results of this study indicated that customized emergency operator assistance could eliminate most user errors observed with using the Schiller FRED EasyPort.

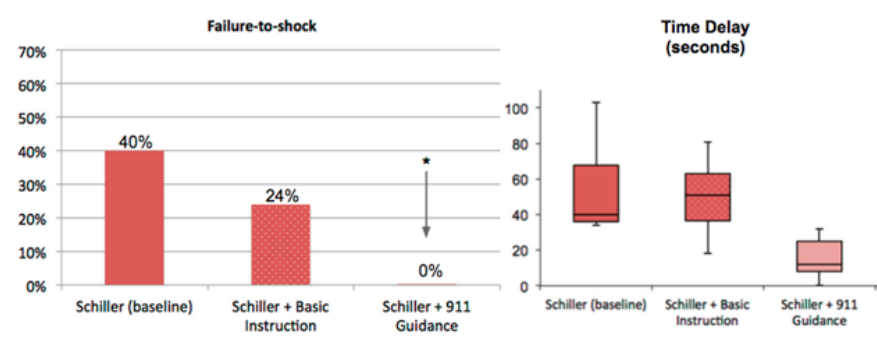

Figure 4. Effect of basic instruction and operator guidance on success rates and time-to-shock.

\section{Discussion}

Defibrillation is a complicated task, with many opportunities for untrained users to fail. Even in a controlled, calm lab scenario, the two tested lightweight AED models yielded multiple errors in unassisted studies. Specific device characteristics (e.g., nonattached electrodes, lack of task-linked instructional cues, ambiguous pictorial and audio cues) drove the majority of observed issues. In reviewing existing literature and feature sets of other devices, it appears that other AEDs (e.g., Philips HeartStart) may yield better success rates due to improved user affordances such as automatic power-on and clear verbal instructions [4]. However, these other AEDs are much heavier devices, presenting numerous engineering challenges within a UAV-delivery scenario.

In the present studies, I found that providing some simple additional instruction reduced errors and providing customized emergency operator assistance eliminated most errors for the lightest weight defibrillator, the Schiller FRED EasyPort. These data suggest that emergency operators could be more effective by utilizing device-specific scripts after identifying what kind of model is in use.

Unfortunately, feasibility research revealed that additional assistance-based solutions present their own challenges. Providing simple written instruction, for example, on the AED package or on the UAV directly is complicated by the fact that medical device instruction is FDA-regulated and cannot be modified. For customizing emergency scripts, I spoke with several professionals within the emergency community and learned that changing existing protocols is a complex, 
arduous process that can take years, even when the changes are in the best interests of the public.

Ultimately, these data highlighted critical usability concerns for layperson use of lightweight AEDs, which carries implications for deploying these devices within a UAV delivery system. For defibrillators targeting layperson use, manufacturers should continually work to mitigate these observed instruction-based failures. As mentioned before, the Schiller FRED EasyPort does not target layperson use, and tests in this population confirmed the difficulty for an untrained user. The HeartSine AED, a device intended for layperson use, has recently been updated with a new model (samaritan 350P) that aims to provide improved CPR and defibrillation instructions. To optimize layperson usability, defibrillator designers should ensure that devices: clearly instruct the need to remove clothing, automatically power on, give clear guidance on pad placement, pre-attach electrodes to the main unit, and provide plenty of looping, task-triggered audio cues for an emergency operator to hear in order to understand how a caller might be blocked and rapidly assist in helping administer the critical shock.

Lastly, manufacturers will hopefully begin to explore how to integrate medical devices with new emergency response systems, such as UAVs, by optimizing usability and weight (for example, replace a standard 3-year battery with a much smaller, but more frequently maintained 3-week battery) for these unique and potentially transformative technological advances which may not safely accommodate retrofitting existing device designs.

\section{Acknowledgements}

I thank Ricardo Prada for advice on study planning, Leila Takayama for helpful comments, Nasson Boroumand and Natasha Shimuk for data collection, and all the volunteers in these studies for their enthusiastic participation.

\section{References}

1. American Heart Association. CPR Facts and Stats. Retrieved October 7, 2015 from

http://cpr.heart.org/AHAECC/CPRAndECC/AboutCP RFirstAid/CPRFactsAndStats/UCM_475748_CPRFacts-and-Stats.jsp.

2. Anthony D. Andre, A. Dawn B. Jorgenson, Jamie A. Froman, David E. Snyder, Jeanne E. Poole. 2004. Automated external defibrillator use by untrained bystanders: Can the public-use model work? Prehospital Emergency Care 8(3):284-291.

3. P. Eames, P.D. Larsen, D.C. Galletly. 2003. Comparison of ease of use of three automated external defibrillators by untrained lay responders. Resuscitation 58:25-30.

4. R. Fleischhackl, H. Losert, M. Haugk, et al. 2004. Differing operational outcomes with six commercially available automated external defibrillators. Resuscitation 62:167-174.

5. Sudden Cardiac Arrest Foundation. Sudden Cardiac Arrest: A Healthcare Crisis. Retrieved October 7, 2015 from http://www.sca-aware.org/aboutsca\#_edn2.

6. V.N. Mosesso, Jr., A.H. Shapiro, K. Stein, et al. 2010. Effects of AED device features on performance by untrained laypersons. Resuscitation 80:1285-1289. 